\title{
Peptide FLNPDVLDI of heparanase is a novel HLA-A2-restricted CTL epitope and elicits potent immunological antitumor effects in vitro with an 8-branched design
}

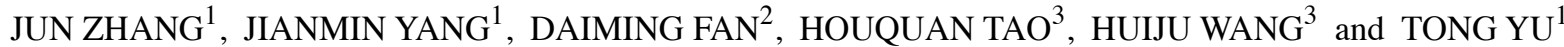 \\ ${ }^{1}$ Department of Gastroenterology, Zhejiang Provincial People's Hospital, Hangzhou, Zhejiang 310014; \\ ${ }^{2}$ State Key Laboratory of Cancer Biology and Institute of Digestive Diseases, Xijing Hospital of Digestive Diseases, \\ Fourth Military Medical University, Xi'an, Shanxi 710032; ${ }^{3}$ Key Laboratory of Gastroenterology \\ of Zhejiang Province, Hangzhou, Zhejiang 310014, P.R. China
}

Received November 22, 2012; Accepted February 20, 2013

DOI: $10.3892 /$ or.2013.2347

\begin{abstract}
The aim of this study was to predict and characterize a novel HLA-A2-restricted T-cell epitope of the human heparanase (HPSE) protein, as well as to evaluate its antitumor immunological effects in vitro with an 8-branched multiple antigenic peptide (MAP) design. The amino acid sequence of HPSE was scanned, and the cytotoxic T lymphocyte (CTL) epitopes were predicted using HLA-A2-restricted epitopepredictive algorithms based on supermotif and quantitative motif methods. The affinity of candidate peptides to HLA-A2 was evaluated using peptide-binding assay, by virtue of the characteristics of T2 cells. The MAPs consisting of the selected peptides were synthesized using an 8-branched design. The specific CTL-inducing ability of the MAPs was assessed by LDH release assay, and the CTL activity was evaluated by INF- $\gamma$ ELISPOT assay. Among the predicted nonapeptides, the FLNPDVLDI peptide of HPSE showed the highest affinity to the HLA-A2 molecule. The 8-branched MAP comprising FLNPDVLDI induced specific CTLs for human HPSE in vitro, which effectively secreted IFN- $\gamma$ and potently lysed HCC97-H human hepatocarcinoma cells and SW-480 human colonic carcinoma cells. The nonapeptide FLNPDVLDI of human HPSE appears to be a novel HLA-A2-restricted CTL epitope, and its 8-branch designed MAP is capable of inducing a potent HPSE-specific CTL response against tumor cells in vitro. Our study provides theoretical evidence for the peptide-based antitumor immunotherapy.
\end{abstract}

Correspondence to: Professor Jianmin Yang, Department of Gastroenterology, Zhejiang Provincial People's Hospital, Hangzhou, Zhejiang 310014, P.R. China

E-mail: zjhealth@tom.com

Key words: heparanase, multiple antigenic peptide, HLA-A2restricted, CTL epitope

\section{Introduction}

A major advance in cancer immunology in the last 2 decades was the verification that the cytotoxic T lymphocyte (CTL) was activated by its epitopes that bind to major histocompatibility complex (MHC) rather than by integral tumor-associated antigens (TAAs) $(1,2)$. Activated CTLs play an important role in the antitumor immunological response by specifically recognizing and subsequently killing tumor cells expressing TAAs $(1,2)$. Dozens of TAAs have been newly identified and well described in recent years (3-6). However, the expression of most TAAs are restricted to a few tumor types and to a fraction of patients with these types of tumors (7-10), and the appearance of antigen-loss or antigen-alteration mutations in tumor cells in response to immune pressure has also been highlighted and extensively elucidated by many researchers (7-10). Many immunotherapeutic trials using vaccinations containing TAAs have been conducted to date, however, clinical trials have demonstrated only limited evidence of tumor regression although a large proportion of patients showed a detectable immune response (11). To circumvent these issues, some researchers advocate that ideal TAAs in tumor cells that are expected to have effective immunological antitumor impacts should be: i) unique, distinctly different than on normal cells; ii) constitutively expressed during the cell cycle; and iii) their constitutive expression is essential for cell survival (12). Thus, a class of TAAs termed 'Universal Tumor Antigens (UTAs) has been proposed that are not only capable of inducing antitumor immunity against a wide range of tumor types, but also play crucially functional roles in tumor growth and development. For years, a vast number of scientific investigations have been carried out to seek promising UTAs before the identification of heparanase (HPSE) epitopes.

The HPSE mRNA encodes a $61.2-\mathrm{kDa}$ protein with 543 amino acids. This pro-enzyme is cleaved into 8 and $50 \mathrm{kDa}$ subunits post-translation and is non-covalently associated to form the active HPSE (13). HPSE is an endoglycosidase that specifically cleaves heparin sulfate (HS) side chains of 
HS proteoglycans, which is the major proteoglycans in the extracellular matrix and cell surfaces (14). HPSE is expressed at a low level in the cells of immune tissues and organs such as the placenta, spleen, thymus and lymph nodes, without expression in other mature tissues. Its overexpression is ubiquitously found in various malignant cell lines and metastatic tumor cells, and is correlated with invasion and metastasis of tumors (15-17). As outlined above, certain tumor cells can downregulate or mutate TAA expression to evade immune surveillance (antigen-loss or antigen-alteration mutations). Nevertheless, because of the crucial role of HPSE in tumor progression, downregulation or mutation of HPSE expression as a means of immune escape probably itself regresses on the proliferation and metastasis of tumor cells. Therefore, HPSE could be considered to be an ideal TAA as well as an UTA in treating human cancers, and the identification of T-cell epitopes of human HPSE have recently been a hot topic in the field of immunotherapy (18). In the present study, we scanned the amino acid sequences of HPSE with prediction softwares SYFPEITHI and BIMAS for possible HPSE-specific CTL epitopes, using HLA-A2-restricted epitope prediction algorithms based on supermotif and quantitative motif methods, and then synthesized an 8-branched multiple antigenic peptide (MAP). Subsequently, peptide binding test, cytotoxicity test and IFN- $\gamma$ secretion assay were carried out. We identified a novel HLA-A2-restricted CTL epitope of HPSE, the MAP of which is capable of inducing an HPSE-specific CTL response against tumor cells in vitro.

\section{Materials and methods}

Cells and cell culture. The human transporter associated with antigen-processing (TAP)-deficient T2 cell line was supplied by the Chinese Center for Disease Control and Prevention (Beijing, China). The human hepatocarcinoma cell line HCC97-H ( $\mathrm{HPSE}^{+}$, HLA-A2 ${ }^{+}$) was purchased from the Liver Cancer Institute, Zhongshan Hospital, Fudan University (Shanghai, China). The human colon cancer cell line SW480 $\left(\mathrm{HPSE}^{+}, \mathrm{HLA}-\mathrm{A} 2^{+}\right)$was obtained from the central laboratory of the Sir Run Run Shaw Hospital, Zhejiang University (Hangzhou, China). T2 and SW480 cells were cultured in RPMI-1640 medium containing $10 \% \mathrm{FBS}$, penicillin (100 U/ml) and streptomycin $(100 \mu \mathrm{g} / \mathrm{ml})$. HCC 97-H cells were maintained in DMEM containing $10 \% \mathrm{FBS}$, penicillin $(100 \mathrm{U} / \mathrm{ml})$ and streptomycin $(100 \mathrm{pg} / \mathrm{ml})$. All cells mentioned above were kept at $37^{\circ} \mathrm{C}$ in a humidified atmosphere containing $5 \% \mathrm{CO}_{2}$.

Peptide prediction and synthesizing. The amino acid sequence of the 50-kDa subunit (Lys158-Ile543) of human HPSE (GenBank accession NP_006656.2) was as follows: KKFKNS TYSRSSVDVLYTFANCSGLDLIFGLNALLRTADLQWN SNAQLLLDYCSSKGYNISWELGNEPNSFLKKADIFIN GSQLGEDFIQLHKLLRKSTFKNAKLYGPDVGQPRRKT AKMLKSFLKAGGEVIDSVTWHHYYLNGRTATKEDF LNPDVLDIFISSVQKVFQVVESTRPGKKVWLGETSSA YGGGAPLLSDTFAAGFMWLDKLGLSARMGIEVVMR QVFFGAGNYHLVDENFDPLPDYWLSLLFKKLVGTKV LMASVQGSKRRKLRVYLHCTNTDNPRYKEGDLTLYA INLHNVTKYLRLPYPFSNKQVDKYLLRPLGPHGLLSK
SVQLNGLTLKMVDDQTLPPLMEKPLRPGSSLGLPAFS YSFFVIRNAKVAACI.

The sequence was scanned by the HLA-A2-restricted epitope-predictive algorithms based on supermotif and quantitative motif methods, using the prediction software SYFPEITHI (Institute for Immunology, University of Tübingen, Tübingen, Germany) and BIMAS (Section of Bioinformatics and Molecular Analysis, National Institutes of Health, Bethesda, MD, USA). Peptides scored high by both SYFPEITHI and BIMAS were selected as candidate epitopes for further evaluation. The predicted peptides were synthesized using Fmoc-Chemistry, and the MAP polypeptides were synthesized with the Solid-Phase Peptide Synthesizer by adopting an 8-branched-peptide design by Chinese Peptide Co. (Hangzhou, China). The peptides and the MAPs were purified using reverse-phase high-performance liquid chromatography (HPLC) on a Vydac C18 column. The purity of the peptides/MAPs was confirmed by analytic HPLC. Lyophilized peptides and MAPs were dissolved to a final concentration of $10 \mathrm{mg} / \mathrm{ml}$ in DMSO and stored at $-80^{\circ} \mathrm{C}$ before use.

Peptide binding test. The binding affinity of the candidate peptides to HLA-A2 was determined by measuring the peptide-induced expression of HLA-A2 molecules on T2 cells using flow cytometry (FCM) (EPICS-XL, Beckman Coulter, Brea, CA USA). Briefly, T2 cells $\left(10^{6} / \mathrm{ml}\right)$ were incubated together with $10 \mu \mathrm{g} / \mathrm{ml}$ of each nonapeptide in serum-free RPMI- 1640 at $37^{\circ} \mathrm{C}$ for $4 \mathrm{~h}$. Consequently, they were washed 3 times with cold PBS to remove free peptides and were incubated with FITC-conjugated antiHLA-A2 monoclonal antibody (mAb) BB7.2 (Santa Cruz Biotechnology, Santa Cruz, CA, USA) at room temperature for $30 \mathrm{~min}$. Subsequently, cells were rinsed 3 times with PBS and were analyzed by FCM. The mean fluorescence index (MFI) was measured, and the fluorescence index (FI) was calculated using the following formula:

$$
\mathrm{FI}=\frac{\text { MFIS }- \text { MFIB }}{\text { MFIB }}
$$

In this formula, MFIS is the MFI of the cell sample, and MFIB is the MFI of the background, representing the MFI value without the nonapeptides. FI $>1.5$ was considered high binding affinity, $1.0<$ FI $<1.5$ was considered intermediate binding affinity and FI $<1.0$ was considered low binding affinity.

CTL generation. Peripheral blood mononuclear cells (PBMCs), obtained from a healthy HLA-A2-positive (determined by the Zhejiang Provincial Blood Center, China) donor, were separated by gradient centrifugation using FicollHypaque Human Lymphocyte Separating solution (Cedarlane Laboratories, Hornby, Ontario, Canada). The PBMCs were then cultured in RPMI-1640 supplemented with 10\% FBS, penicillin $(100 \mathrm{U} / \mathrm{ml})$ and streptomycin $(100 \mu \mathrm{g} / \mathrm{ml})$, and were stimulated by the synthetic MAP polypeptides $(10 \mu \mathrm{g} / \mathrm{ml})$ the following day. The PBMCs were re-stimulated every 7 days with fresh medium containing these MAPs. Recombinant interleukin $2(100 \mathrm{U} / \mathrm{ml})$ (More Bio Co., Ltd., China) was added to the culture media $24 \mathrm{~h}$ after every stimulation. The CTLs were thus harvested and the activity was assessed on day 23. 
Cytotoxicity assay. The cytotoxic activity of the CTLs was evaluated by lactate dehydrogenase (LDH) release assay (Promega, Madison, WI, USA). Human hepatocellular carcinoma cell line HCC97-H and human colorectal cancer cell line SW480 served as target cells. The cells $\left(10^{6} / \mathrm{ml}\right)$ were incubated with the candidate MAP polypeptides $(10 \mu \mathrm{g} / \mathrm{ml})$ for $4 \mathrm{~h}$ at $37^{\circ} \mathrm{C}$. Then, the HCC $97-\mathrm{H}$ and SW480 cells were collected, washed with PBS and resuspended at a concentration of $10^{5} / \mathrm{ml}$ and $2 \times 10^{5} / \mathrm{ml}$, respectively (according to the result of our pre-experiment on optimizing the amount of target cells), in a phenol red-free media containing 5\% FBS. Subsequently, a 50- $\mu$ l cell suspension was plated into each well of a 96-well round-bottomed plate, and mixed with effector cells $(50 \mu \mathrm{l} /$ well) at a 40:1, 20:1 or 10:1 effector to target (E/T) ratio. Assays were conducted in triplicate for each sample at each ratio. After incubation for $4 \mathrm{~h}$, the supernatants were harvested. Absorbance at $490 \mathrm{~nm}$ was measured in triplicate using a microplate reader (BioTek, Richmond, VA, USA), and the cytotoxicity was calculated according to the following formula:

$$
\text { Cytotoxicity }(\%)=\frac{(\mathrm{LDH}) \mathrm{E}-(\mathrm{LDH}) \mathrm{ES}-(\mathrm{LDH}) \mathrm{TS}}{(\mathrm{LDH}) \mathrm{TSmax}-(\mathrm{LDH}) \mathrm{TS}} \times 100 \%
$$

Here, (LDH)E is experimental LDH release, (LDH)ES is the effector spontaneous $\mathrm{LDH}$ release, $(\mathrm{LDH}) \mathrm{TS}$ is the target spontaneous LDH release and (LDH)TSmax is the target maximum LDH release. Spontaneous LDH release was assessed by incubating target cells free of effector cells, or effector cells lacking target cells, while maximum release was determined by the presence of effector cells.

Enzyme-linked immunospot assay for IFN- $\gamma$. The generated specific CTLs were investigated using an enzyme-linked immunospot (ELISPOT) kit (U-Cytech Biosciences, Utrecht, The Netherlands) for INF- $\gamma$ production. Briefly, effectors were plated in triplicate at a final concentration of $2.5 \times 10^{5}$ cells/well in 96-well nitrocellulose plates. Effectors were stimulated by candidate MAPs and at a final concentration of $30 \mu \mathrm{mol} / 1$ and by PBS as a negative control. Afterwards, the plate was incubated at $37^{\circ} \mathrm{C}$ in $5 \% \mathrm{CO}_{2}$ for $24 \mathrm{~h}$. The plate was processed using a biotin-labeled anti-mouse INF- $\gamma$, enzyme-labeling marker and anti-marker at $37^{\circ} \mathrm{C}$ for $1 \mathrm{~h}$. Then, the freshly prepared developer was added and incubated in the dark at $37^{\circ} \mathrm{C}$ for $25 \mathrm{~min}$. Spots were quantified using the ELISPOT reader (BioReader 4000 Pro-X, Biosys).

Statistical analysis. All experiments were carried out in triplicate, and the data are represented as the means \pm SD of triplicate determinations. Statistical analysis was conducted using the Student's t-test or variance analysis. Differences were considered statistically significance at a P-value $<0.05$. All statistical analyses were conducted using SPSS 11.5 software (SPSS Inc., Chicago, IL, USA).

\section{Results}

Screening and identification of potential HLA-A2-restricted HPSE epitopes. To select the candidate HLA-A2-restricted epitopes, the amino acid sequence of HPSE was screened to predict the HLA-A2 binding motif, using SYFPEITHI

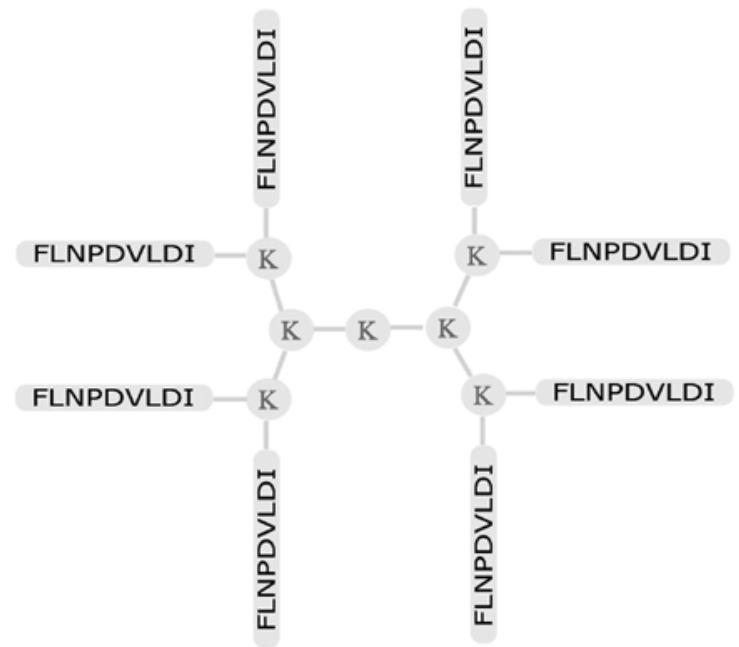

Figure 1. Schematic structure of the synthesized multiple antigenic peptides (MAP) based on the predicted CTL epitope of human heparanase (HPSE). Based on the amino acid sequence of HPSE and the predicted CTL epitope, MAP was synthesized with the Solid-Phase Peptide Synthesizer by adopting an 8-branched peptide design. Eight peptides were interconnected by lys (K).

together with BIMAS as outlined above. As a result, 6 HPSE nonapeptides were selected with high binding scores in both SYFPEITHI and BIMAS programs: peptide LIFGLNALL (184-192), peptide FLNPDVLDI (310-318), peptide VLDIFISSV (315-323), peptide FMWLDKLGL (363-371), peptide WLSLLFKKL (405-413) and peptide GLLSKSVQL (487-495), as shown in Table I.

Peptide FLNPDVLDI has the highest binding affinity to $H L A-A 2$. According to the prediction outcomes, the candidate peptides and the 8-branched MAP polypeptides (Fig. 1) were synthesized and purified. The purities of the peptides and MAPs reached $95 \%$ or more by purification using HPLC. To investigate the binding affinity of these peptides to the HLA-A 2 molecule, a T2 cell peptide binding assay was carried out, by which the upregulation of HLA-A2 molecules on T2 cells followed by the exposure to exogenous HLA-A2 binding peptides could be measured. The FI of peptide LIFGLNALL (184-192), peptide FLNPDVLDI (310-318), peptide VLDIFISSV (315-323), peptide FMWLDKLGL (363-371), peptide WLSLLFKKL (405-413) and peptide GLLSKSVQL (487-495) was 1.16, 1.70, 1.51, 1.05, 0.79 and 0.76 , respectively (Table II). Of these 6 nonapeptides, HPSE (310-318) and HPSE (315-323) showed high binding affinities to HLA-A2 molecules (FI $>1.5$ ), while others showed low or moderate affinities (FI $<1.0$ or $1.0<\mathrm{FI}<1.5)$. Among them, the FI of peptide FLNPDVLDI (310-318) was the highest (FI=1.70), which appeared to be the HLA-A2- restricted CTL epitope of HPSE.

Cytotoxic activity of the CTL cells. Human PBMCs were stimulated by the synthesized MAP polypeptides. The cytotoxic activity of the CTLs generated from the PBMCs was measured by LDH release assay. Among these 6 MAP polypeptides, the MAP consisting of FLNPDVLDI induced a potent peptidespecific CTL cytotoxicity against both HCC97-H (HLA-A2+, 
Table I. HLA-A2-restricted CTL epitopes of human heparanase (HPSE) predicted by BIMAS and SYFPEITHI softwares.

\begin{tabular}{llcccc}
\hline Peptide & Sequence & BIMAS score & BIMAS ranking & SYFPEITHI score & SYFPEITHI ranking \\
\hline HPSE (184-192) & LIFGLNALL & 18.476 & 11 & 26 & 4 \\
HPSE (310-318) & FLNPDVLDI & 110.379 & 6 & 27 & 3 \\
HPSE (315-323) & VLDIFISSV & 413.361 & 1 & 28 & 22 \\
HPSE (363-371) & FMWLDKLGL & 589.056 & 3 & 22 & 11 \\
HPSE (405-413) & WLSLLFKKL & 186.707 & 9 & 26 & 5 \\
HPSE (487-495) & GLLSKSVQL & 79.041 & & 5
\end{tabular}

The sequence of HPSE was scanned by HLA-A2-restricted epitope predictive algorithms based on supermotif and quantitative motif methods, using prediction software SYFPEITHI and BIMAS. Peptides showed high scores by both SYFPEITHI and BIMAS were selected as candidate epitopes for further evaluation. As a result, 6 high-score nonapeptides in both SYFPEITHI and BIMAS were chosen as candidates: peptide LIFGLNALL (184-192), peptide FLNPDVLDI (310-318), peptide VLDIFISSV (315-323), peptide FMWLDKLGL (363-371), peptide WLSLLFKKL (405-413) and peptide GLLSKSVQL (487-495).

Table II. Binding affinity of HPSE-derived peptides to the HLA-A2 molecule.

\begin{tabular}{|c|c|c|c|c|}
\hline Peptide & Sequence & HLA-A2 expression (\%) & MFI & FI \\
\hline HPSE (184-192) & LIFGLNALL & 87.8 & 4.21 & 1.16 \\
\hline HPSE (310-318) & FLNPDVLDI & 88.7 & 5.27 & 1.70 \\
\hline HPSE (315-323) & VLDIFISSV & 88.8 & 4.89 & 1.51 \\
\hline HPSE (363-371) & FMWLDKLGL & 86.8 & 3.99 & 1.05 \\
\hline HPSE (405-413) & WLSLLFKKL & 74.6 & 3.50 & 0.79 \\
\hline HPSE (487-495) & GLLSKSVQL & 79.9 & 3.43 & 0.76 \\
\hline Background & - & 39.2 & 1.95 & - \\
\hline
\end{tabular}

T2 cell peptide binding assay was performed to investigate the binding affinity of the candidate nonapeptides to HLA-A2 molecules. The FI of HPSE (184-192), HPSE (310-318), HPSE (315-323), HPSE (363-371), HPSE (405-413) and HPSE (487-495) was 1.16, 1.70, 1.51, 1.05, 0.79 and 0.76 , respectively. Among them, HPSE (310-318) and HPSE (315-323) showed high binding affinity to HLA-A2 molecules (FI $>1.5)$, while others showed low or moderate affinity $(\mathrm{FI}<1.0$ or $1.0<\mathrm{FI}<1.5)$, and the FI of peptide HPSE $(310-318)$ was the highest $(\mathrm{FI}=1.70)$, which appeared to be the HLA-A2 restricted CTL epitope of HPSE.
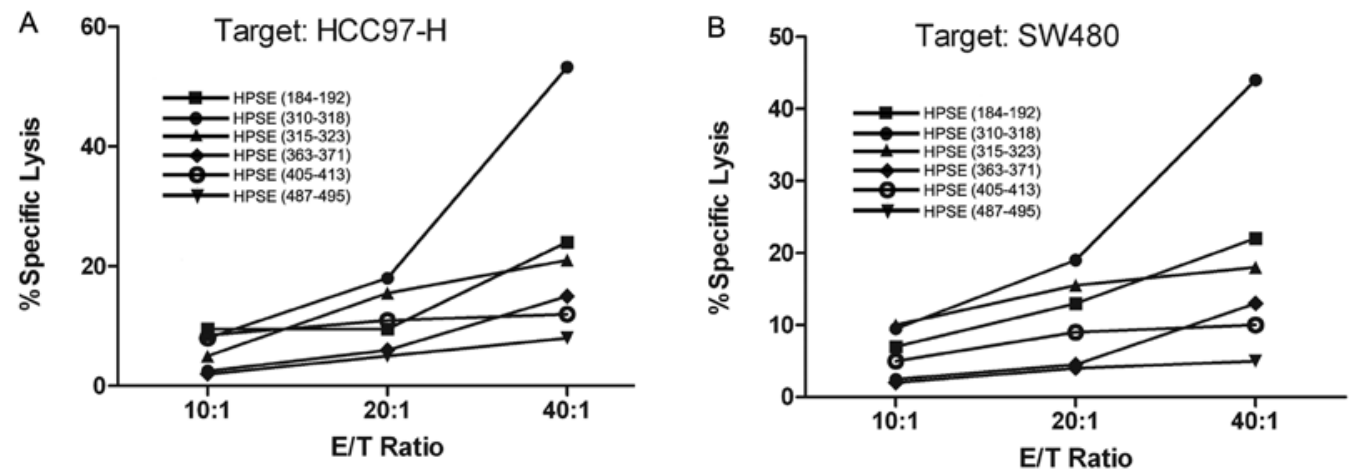

Figure 2. Specific lysis of CTLs towards tumor cells. (A) Specific lysis of CTLs generated from different heparanase (HPSE) peptide-derived multiple antigenic polypeptides (MAPs) against HCC97-H hepatocarcinoma cells in vitro. The MAP consisting of peptide FLNPDVLDI (310-318 of HPSE) effectively induced HLA-A2-restricted and peptide-specific cytotoxicity of CTLs against HCC97-H at an E/T ratio of 40:1, and the specific lysis ratio was 53.21\%. Other MAPs did not induce obvious CTL cytotoxicities. (B) Specific lysis of CTLs generated from different HPSE peptide-derived MAPs against SW480 colonic cancer cells in vitro. Among the MAPs comprising corresponding candidate peptides, MAP-FLNPDVLDI (310-318 of HPSE) was the only peptide that effectively induced HLA-A2-restricted and peptide-specific cytotoxicity of CTLs against SW480 cells at an E/T ratio of 40:1, and the corresponding cytotoxicity ratio was $43.98 \%$. While the specific lysis rates of other MAPs did not exceed $25 \%$ even at the highest E/T ratio.

$\mathrm{HPSE}^{+}$) and SW-480 (HLA-A2 ${ }^{+}, \mathrm{HPSE}^{+}$) tumor cells. At the highest $\mathrm{E} / \mathrm{T}$ ratio $(40: 1)$, the specific lysis rates generated from
MAP-FLNPDVLDI towards HCC97-H hepatocarcinoma cancer cells and SW-480 colonic cancer cells were 53.21 and 


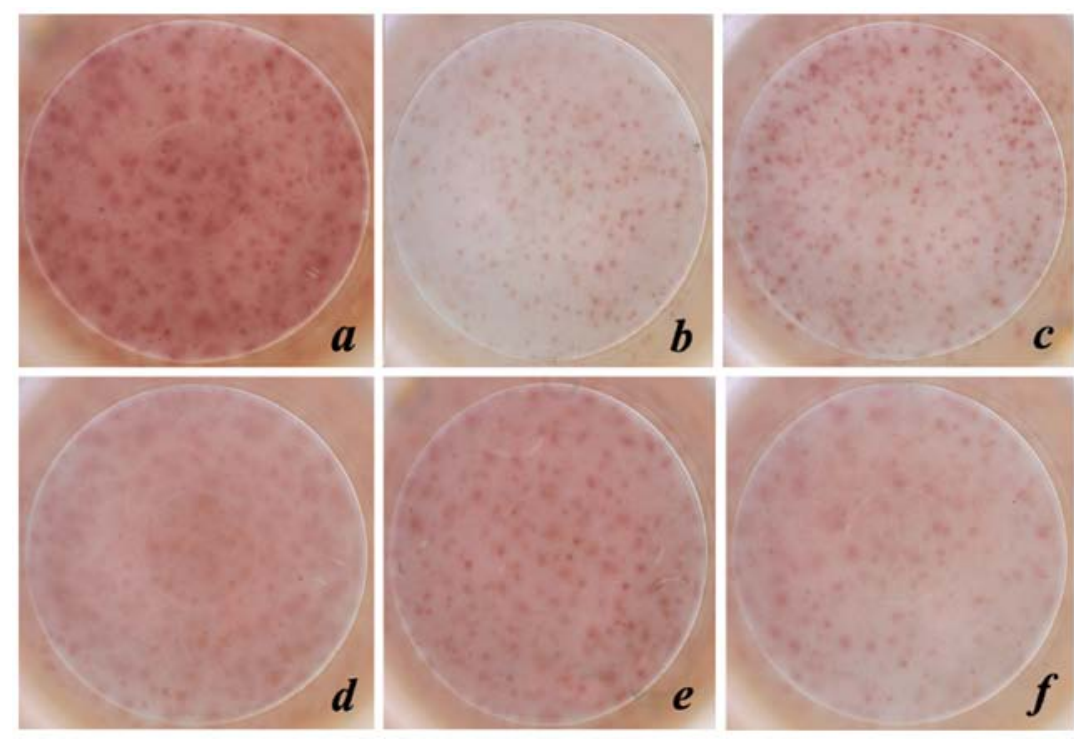

Figure 3. Representative images of immunospots in 96-well nitrocellulose plates. As CTLs are known to produce the Th1 cytokine IFN- $\gamma$, peptide-specific $\mathrm{T}$ cells could be quantitated by measuring IFN- $\gamma$-producing cells using ELISPOT assay. Representative images show the spots induced by the MAPs consisting of (a) peptide FLNPDVLDI (310-318), (b) peptide LIFGLNALL (184-192), (c) peptide VLDIFISSV (315-323), (d) peptide FMWLDKLGL (363-371), (e) peptide WLSLLFKKL (405-413) and (f) peptide GLLSKSVQL (487-495) of HPSE, respectively.

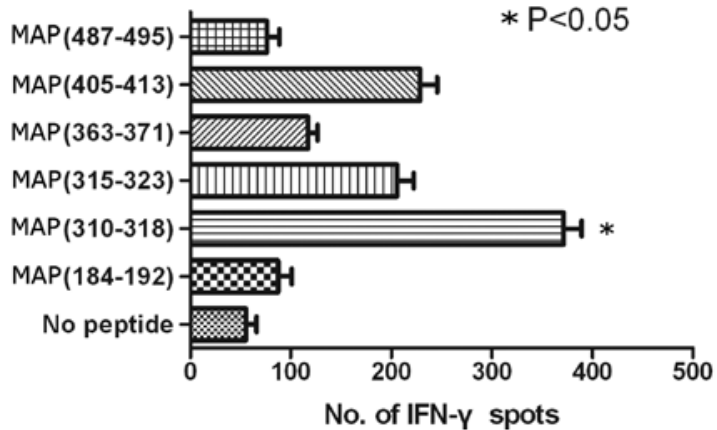

Figure 4. Analysis diagram of the ELISPOT results. The generated specific CTLs were assayed using enzyme-linked immunospot (ELISPOT) for INF- $\gamma$ production. Effectors were stimulated by the 68 -branched multiple antigenic polypeptides (MAPs) and at a final concentration of $30 \mu \mathrm{mol} / 1$ for each, and by PBS (without MAP) as negative control. The average number of spots of the specific CTLs induced by the 8-branch designed MAP-GLLSKSVQL (487-495), MAP-WLSLLFKKL (405-413), MAP-FMWLDKLGL (363-371), MAP-VLDIFISSV (315-323), MAP-FLNPDVLDI (310-318), MAP-LIFGLNALL (184-192) and the negative control was $77 \pm 8,232 \pm 11$, $116 \pm 6,202 \pm 9,371 \pm 12,89 \pm 7$ and $56 \pm 5$, respectively. The number of spots induced by MAP-FLNPDVLDI (310-318 of HPSE) was significantly higher than that of the others induced by the remaining MAPs $\left({ }^{*} \mathrm{P}<0.05\right)$ or the negative control $(\mathrm{P}<0.05)$.

$43.98 \%$, respectively. However, the induced effectors generated from other MAPs did not show obvious lytic potency towards either of the target tumor cells. There were statistical significances between MAP-FLNPDVLDI and other MAP candidates $(\mathrm{P}<0.05)$, by two-factor variance analysis, as shown in Figs. 2 and 3. These results indicate that MAP consisting of peptide FLNPDVLDI was capable of eliciting potent lytic impairments on certain tumor cells in vitro.

ELISPOT assay for IFN- $\gamma$. As CTLs are known to produce the Th1 cytokine IFN- $\gamma$, peptide-specific T cells can be quantitated by measuring IFN- $\gamma$-producing cells using ELISPOT assay. In this experiment, the spots number induced by the MAP-FLNPDVLDI was $371 \pm 12$, which generated significantly stronger peptide-specific T-cell responses by virtue of its ability to induce increased frequencies of IFN- $\gamma$-producing $\mathrm{T}$ cells, as compared with the negative control and other candidate MAPs $(\mathrm{P}<0.05)$. The representative images of immunospots in nitrocellulose plates are shown in Fig. 3, and the analysis of this experiment is illustrated in Fig. 4.

\section{Discussion}

To date, conventional therapies for cancer include surgery, chemotherapy, and radiotherapy as well as the combination of these methods (19). However, malignant tumors consistently respond poorly to these treatments, and remnant tumor cells lead to recurrence after surgery. Traditional chemotherapy and radiation therapy harm normal cells at the same time they kill malignant cells. Therefore, novel therapeutic strategies are urgently needed for the treatment of these diseases. Tumor immunotherapy is a feasible treatment that can lower the rate of relapse and improve the recurrence-free outcomes after surgery (20), afflicting less side effects than both chemotherapy and radiation (20). Over the past 2 decades, several immunotherapeutic approaches have been developed, such as dendritic cell-based vaccines, DNA-based vaccines and protein-based vaccines (21-23). However, there are still many setbacks to some of these immunotherapies as mentioned previously (7-11).

CTLs are considered essential to the anticancer immunoresponse, and immunotherapeutic approaches using synthetic peptides based on CTL epitopes have been under intense investigation recently. CTL epitopes have many advantages. They are easy to be produced even at clinical grade, free from pathogen contamination, and have low oncogenic potential and high chemical stability (24). Peptides derived from HLA-A2-restricted CTL epitopes of TAAs are proven to be 
specifically recognized by CTLs (25), induce potent antitumor CTL responses and result in growth regression of tumors and prevention of the formation metastasis $(25,26)$. In this regard, although in its infancy, the field of immunotherapeutics based on CTL-inducing epitopes is rapidly approaching the point at which crucial data will be gathered regarding its potential in cancer immunotherapy.

HPSE is an important cancer-associated enzyme. HPSE has been detected at relatively low levels in mammalian immune tissues and organs, such as the placenta, spleen, thymus and lymph node, and it is either not expressed or expressed at very low levels in other normal tissues (27). However, HPSE was found to be highly expressed in most mammalian malignant tumors, and the increased expression and secretion of HPSE by tumor cells promote tumor invasion by extracellular matrix (ECM) degradation, tissue destruction, angiogenesis and metastasis (15). Therefore, prediction and identification of HLA-A2-restricted CTL epitopes of HPSE are very promising in exploiting a new peptide vaccine for anticancer treatment. The first group of immunogenic CTL epitopes in HPSE was discovered by Sommerfeldt et al in 2006 (28), using the SYFPEITHI algorithm to identify nonapeptides of the HPSE amino acid sequence.

Although the development of anticancer vaccines by applying the CTL epitope of TAA is expected to be promising, the immunogenicity of epitope peptide is poor, and an ideal immune response usually cannot be induced due to its small molecular weight and single structure (29). Traditionally, the epitope peptide crosslinking carrier protein method was used to improve the immunogenicity of epitope peptides, and in recent years, a MAP design plan was proposed by some researchers (30). The lysine core matrix of small molecular weight and weak immunogenicity was taken to couple a number of monomeric peptides and to form a dendritic structure, and this 8-branched design was also successfully used in synthesizing the MAPs in this study.

In the present study, we identified 6 nonapeptides by HLA-A2-restricted epitope predictive algorithms based on supermotif and quantitative motif methods, making use of the predictive softwares SYFPEITHI and BIMAS. According to the principle of supermotif theoretics (31), the affinity between peptide and HLA-A 2 would be relatively higher if the 2nd and the 9th amino acids are among the following list: $\mathrm{L}, \mathrm{I}, \mathrm{V}, \mathrm{M}, \mathrm{A}, \mathrm{T}$, and $\mathrm{Q}$ (32), which are perfectly encompassed in our 6 candidate peptides. Of the 6 nonapeptides, peptide LIFGLNALL (184-192) of HPSE has the least difference from the epitope peptide DLIFGLNAL (183-191) of HPSE identified by Sommerfeldt et al (28). However, an effective CTL response induced by epitope peptide LIFGLNALL was not detected in our investigation, which was considered capable of lysing BT-20, BT-549 and BT-124 mammary cancer cells by Sommerfeldt and co-workers (28). One reasonable interpretation for this contradictory outcome was that tiny disparities in residue sequence may lead to huge changes in peptide constellation, which is most essential in immunogenicity. Another candidate nonapeptide WLSLLFKKL (405-413) of HPSE is completely consistent with the CTL epitope predicted by Tang et al (33) and Chen et al (34). Nevertheless, its corresponding MAP polypeptide was not able to elicit the specific CTL response and subsequently lyse tumor cells in our experiment, which are completely dissimilar to these authors observations using KATOIII gastric cancer cells and measured by ${ }^{51} \mathrm{Cr}$ release assay. The variation in tumor cell targets and the difference in detecting techniques of the CTL activity might be responsible for these inconsistencies. We used the LDH release assay in this study to determine the cytotoxicity of CTLs againt tumor cells. Although LDH assay has a much higher specificity than the ${ }^{51} \mathrm{Cr}$ release assay, it is less sensitive than the latter (35). Thereby, the inefficiency of the epitope peptide WLSLLFKKL here may be just a false-negative result due to the relatively lower sensitivity of the LDH release assay itself. By generalizing the outcomes of our experimental results for the peptide binding test, $\mathrm{LDH}$ release assay and IFN- $\gamma$ ELISPOT assay, the nonapeptide FLNPDVLDI of HPSE performed most excellently among the 6 candidates. It had the highest affinity to the HLA-A2 molecule, and its corresponding MAP polypeptide effectively induced the generation of peptide-specific CTLs, which can secret IFN- $\gamma$ effectively and have strong lytic cytotoxicity on HCC97-H and SW480 cells.

In summary, of the 6 predicted nonapeptides, eiptope peptide FLNPDVLDI (310-318) of human HPSE probably is a novel HLA-A2-restricted CTL epitope, and the MAP consisting of peptide FLNPDVLDI aroused a specific CTL response in vitro, which was lethal to tumor cells expressing both HPSE and HLA-A2. Therefore, this study provides theoretical evidence for the further study of MAP vaccines based on CTL epitopes of HPSE in cancer immunotherapies.

\section{Acknowledgements}

This study was supported by the National Natural Science Foundation of China (no. 30570816).

\section{References}

1. Gilboa E: The makings of a tumor rejection antigen. Immunity 11: 263-270, 1999.

2. Boon T, Cerottini JC, Van den Eynde B, et al: Tumor antigens recognized by T lymphocytes. Annu Rev Immunol 12: 337-365, 1994.

3. Chakraborty NG, Stevens RL, Mehrotra S, et al: Recognition of PSA-derived peptide antigens by $\mathrm{T}$ cells from prostate cancer patients without any prior stimulation. Cancer Immunol Immunother 52: 497-505, 2003.

4. Nakatsugawa M, Horie K, Yoshikawa T, et al: Identification of an HLA-A*0201-restricted cytotoxic T lymphocyte epitope from the lung carcinoma antigen, Lengsin. Int J Oncol 39: 1041-1049, 2011.

5. Fleischhauer K, Gattinoni L, Lietti G, et al: Identification of tumor antigen-specific cytotoxic $\mathrm{T}$ lymphocytes cross-recognizing allogeneic major histocompatibility class I molecules. Tissue Antigens 56: 19-29, 2000.

6. Yokokawa J, Bera TK, Palena C, et al: Identification of cytotoxic T-lymphocyte epitope(s) and its agonist epitope(s) of a novel target for vaccine therapy (PAGE4). Int J Cancer 121: 595-605, 2007.

7. Rabinovich GA, Gabrilovich D and Sotomayor EM: Immunosuppressive strategies that are mediated by tumor cells. Annu Rev Immunol 25: 267-296, 2007.

8. Ostrand-Rosenberg S: Immune surveillance: a balance between protumor and antitumor immunity. Curr Opin Genet Dev 18: 11-18, 2008.

9. Somasundaram R, Jacob L, Swoboda R, et al: Inhibition of cytolytic T lymphocyte proliferation by autologous $\mathrm{CD} 4^{+} / \mathrm{CD} 25^{+}$ regulatory $\mathrm{T}$ cells in a colorectal carcinoma patient is mediated by transforming growth factor- $\beta$. Cancer Res 62: 5267-5272, 2002. 
10. Kolar P, Knieke K, Hegel JK, et al: CTLA-4 (CD152) controls homeostasis and suppressive capacity of regulatory $\mathrm{T}$ cells in mice. Arthritis Rheum 60: 123-132, 2009.

11. Zarour HM and Ferrone S: Cancer immunotherapy: progress and challenges in the clinical setting. Eur J Immunol 41: 1510-1515, 2011.

12. Darzynkiewicz Z: Will cancer immunotherapy fail? Scientist 20: 14, 2006.

13. McKenzie E, Young K, Hircock M, et al: Biochemical characterization of the active heterodimer form of human heparanase (Hpa1) protein expressed in insect cells. Biochem J 373: 423-435, 2003.

14. McKenzie EA: Heparanase: a target for drug discovery in cancer and inflammation. Br J Pharmacol 151: 1-14, 2007.

15. Cohen I, Pappo O, Elkin M, et al: Heparanase promotes growth, angiogenesis and survival of primary breast tumors. Int J Cancer 118: 1609-1617, 2006.

16. Shafat I, Zcharia E, Nisman B, et al: An ELISA method for the detection and quantification of human heparanase. Biochem Biophys Res Commun 341: 958-963, 2006.

17. Wang $\mathrm{Z}, \mathrm{Xu} \mathrm{H}$, Jiang L, et al: Positive association of heparanase expression with tumor invasion and lymphatic metastasis in gastric carcinoma. Mod Pathol 18: 205-211, 2005.

18. Zhang YF, Tang XD, Gao JH, et al: Heparanase: a universal immunotherapeutic target in human cancers. Drug Discov Today 16: 412-417, 2011

19. Ritter M, Ali MY, Grimm CF, et al: Immunoregulation of dendritic and $\mathrm{T}$ cells by alpha-fetoprotein in patients with hepatocellular carcinoma. J Hepatol 41: 999-1007, 2004

20. Borghaei H, Smith MR and Campbell KS: Immunotherapy of cancer. Eur J Pharmacol 625: 41-54, 2009.

21. Lemoine FM, Cherai M, Giverne C, et al: Massive expansion of regulatory $\mathrm{T}$-cells following interleukin 2 treatment during a phase I-II dendritic cell-based immunotherapy of metastatic renal cancer. Int J Oncol 35: 569-581, 2009.

22. Williams BB, Wall M, Miao RY, et al: Induction of T cell-mediated immunity using a c-Myb DNA vaccine in a mouse model of colon cancer. Cancer Immunol Immunother 57: 1635-1645, 2008

23. Viehl CT, Becker-Hapak M, Lewis JS, et al: A tat fusion proteinbased tumor vaccine for breast cancer. Ann Surg Oncol 12: $517-525,2005$
24. van der Burg SH, Bijker MS, Welters MJ, et al: Improved peptide vaccine strategies, creating synthetic artificial infections to maximize immune efficacy. Adv Drug Deliv Rev 58: 916-930, 2006.

25. Horiguchi Y, Nukaya I, Okazawa K, et al: Screening of HLA-A24-restricted epitope peptides from prostate-specific membrane antigen that induce specific antitumor cytotoxic $\mathrm{T}$ lymphocytes. Clin Cancer Res 8: 3885-3892, 2002.

26. Feuerer M, Beckhove P, Bai L, et al: Therapy of human tumors in NOD/SCID mice with patient-derived reactivated memory $\mathrm{T}$ cells from bone marrow. Nat Med 7: 452-458, 2001.

27. Zcharia E, Metzger S, Chajek-Shaul T, et al: Molecular properties and involvement of heparanase in cancer progression and mammary gland morphogenesis. J Mammary Gland Biol Neoplasia 6: 311-322, 2001.

28. Sommerfeldt N, Beckhove P, Ge Y, et al: Heparanase: a new metastasis-associated antigen recognized in breast cancer patients by spontaneously induced memory $\mathrm{T}$ lymphocytes. Cancer Res 66: 7716-7723, 2006.

29. Zarour HM and Ferrone S: Cancer immunotherapy: progress and challenges in the clinical setting. Eur J Immunol 41: 1510-1516, 2011.

30. Liu X, Guo J, Han S, et al: Enhanced immune response induced by a potential influenza A vaccine based on branched M2e polypeptides linked to tuftsin. Vaccine 30: 6527-6533, 2012.

31. Sidney J, Grey HM, Kubo RT, et al: Practical, biochemical and evolutionary implications of the discovery of HLA class I supermotifs. Immunol Today 17: 261-266, 1996.

32. Falk K, Rotzschke O, Stevanovic S, et al: Allele-specific motifs revealed by sequencing of self-peptides eluted from MHC molecules. Nature 351: 290-296, 1991.

33. Tang XD, Liang GP, Li C, et al: Cytotoxic T ymphocyte epitopes from human heparanase can elicit a potent anti-tumor immune response in mice. Cancer Immunol Immunother 59: 1041-1047, 2010.

34. Chen T, Tang XD, Wan Y, et al: HLA-A2-restricted cytotoxic T lymphocyte epitopes from human heparanase as novel targets for broad-spectrum tumor immunotherapy. Neoplasia 10: 977-986, 2008.

35. Yokozawa T, Miyarnura K, Fujino R, et al: Gelatin beads as platforms for targeting molecule and anti-Fas antibody: two major properties of cytotoxic T lymphocytes. Exp Hematol 28: 1129-1136, 2000 